\title{
Long-Term Cognitive Changes after Revascularization Surgery in Adult Patients with Ischemic Moyamoya Disease
}

\author{
Shun Uchida ${ }^{a}$ Yoshitaka Kubo $^{a}$ Daisuke Oomori ${ }^{a}$ Masahiro Yabuki ${ }^{a}$ \\ Kei Kitakamia Shunrou Fujiwara ${ }^{a}$ Kenji Yoshida ${ }^{a}$ Masakazu Kobayashia \\ Kazunori Terasaki ${ }^{\text {b }}$ Kuniaki Ogasawara ${ }^{a}$ \\ aDepartment of Neurosurgery, Iwate Medical University School of Medicine, Yahaba, Japan; 'blnstitute for Biomedical \\ Sciences, Iwate Medical University School of Medicine, Yahaba, Japan
}

\section{Keywords}

Moyamoya disease $\cdot$ Adult · Ischemia $\cdot$ Revascularization ·

Cognition

\begin{abstract}
Introduction: Revascularization surgery for adult moyamoya disease (MMD) with ischemic presentation changes cognitive function and prevents further cerebral ischemic events. Most studies however repeated neuropsychological evaluation within 1 year after surgery. Our previous prospective cohort study of adult patients with MMD with misery perfusion who underwent direct revascularization surgery showed cognitive improvement and decline in 31\% and $44 \%$, respectively, of the patients 2 months after surgery. The present prospective study aimed to elucidate the 5-year cognitive changes after direct revascularization surgery in adult patients with cerebral misery perfusion due to ischemic MMD by following the same patients. Methods: In total, 31 patients were prospectively followed up for 5 years after direct revascularization surgery. Five types of neuropsychological tests were performed preoperatively, 2 months after surgery, and at the end of the 5-year follow-up. Cerebral blood flow (CBF) in the symptomatic cerebral hemisphere
\end{abstract}

karger@karger.com www.karger.com/cee

Karger!"
(C) 2021 The Author(s)

Published by S. Karger AG, Basel

This is an Open Access article licensed under the Creative Commons Attribution-NonCommercial-4.0 International License (CC BY-NC) (http://www.karger.com/Services/OpenAccessLicense), applicable to the online version of the article only. Usage and distribution for commercial purposes requires written permission. relative to that in the ipsilateral cerebellar hemisphere (hemispheric relative $\mathrm{CBF}[\mathrm{RCBF}]$ ) was measured using brain perfusion single-photon emission computed tomography preoperatively and at the end of the 5-year follow-up. Results: Based on results of pre- and postoperative neuropsychological tests, 11, 10, and 10 patients showed cognitive improvement, no change in cognitive function, and cognitive decline, respectively, at the end of the 5-year follow-up. These ratios were not significantly different compared with those 2 months after surgery (cognitive improvement, no change in cognitive function, and cognitive decline in 10, 8, and 13 patients, respectively). Although hemispheric RCBF was significantly greater at the end of the 5-year follow-up than before surgery in patients with cognitive improvement $(80.7 \pm$ $6.1 \%$ vs. $92.9 \pm 5.5 \% ; p=0.0033$ ) and in those showing no change in cognitive function $(85.6 \pm 3.5$ vs. $91.5 \pm 5.2 \%$; $p=$ $0.0093)$, this value was significantly lower at the end of the 5 -year follow-up than before surgery in patients with cognitive decline ( $83.8 \pm 3.7$ vs. $81.0 \pm 5.8 \% ; p=0.0367)$. Conclusion: One-third of adult patients with cerebral misery perfusion due to ischemic MMD who underwent direct revascularization surgery exhibited cognitive improvement, and one-third exhibited decline at the end of the 5-year followup. The former and latter patients had increased and de- 
creased $\mathrm{CBF}$, respectively, in the affected cerebral hemisphere at the end of the 5-year follow-up compared with preoperative brain perfusion. @ 2021 The Author(s).

Published by S. Karger AG, Basel patients with cerebral misery perfusion due to ischemic MMD by following up previously reported patients [10] for 5 years.

\section{Materials and Methods}

\section{Introduction}

Moyamoya disease (MMD) is characterized by chronic bilateral steno-occlusive changes in the terminal portion of the internal carotid artery and abnormal arterial collateral flow at the base of the brain $[1,2]$. For adult patients with ischemic symptoms due to MMD, strict medical management alone including an antiplatelet drug such as cilostazol may be recommended when the symptomatic cerebral hemisphere exhibits stable hemodynamics [3-5]. In contrast, when the symptomatic cerebral hemisphere exhibits hemodynamic compromise such as misery perfusion, typical treatment is direct revascularization surgery, such as anastomosis of the superficial temporal artery to the middle cerebral artery, with or without indirect revascularization surgery, such as encephalo-duro-myo-synangiosis [3, 4, 6-10].

Revascularization surgery in adult MMD changes cognitive function and prevents further cerebral ischemic events [6,11-15]. Most adult MMD patients who undergo revascularization surgery do not show cognitive changes relative to cognitive ability before surgery, although some patients do demonstrate improved or worsened cognitive function postoperatively [16]. We previously carried out a prospective study in which adult patients with ischemic MMD who had misery perfusion in the symptomatic cerebral hemisphere underwent direct revascularization surgery and neuropsychological testing before and after surgery [10]. This study showed that $31 \%$ and $44 \%$ of the patients had postoperative cognitive improvement and decline, respectively [10]. Immediately after direct revascularization surgery, cerebral hyperperfusion leads to cognitive dysfunction [10]. In contrast, a gradual increase in cerebral blood flow (CBF) in later stages leads to improvements in cognitive function in the absence of early-stage cerebral hyperperfusion [10].

Most of these studies however repeated neuropsychological evaluation within 1 year after surgery $[10,11,13-$ $16]$. Only 1 study performed neuropsychological testing $>1$ year but $<2$ years postoperatively [12]. Longer term cognitive changes after revascularization surgery for adult MMD patients remain uninvestigated. The present prospective study aimed to elucidate the 5-year cognitive changes after direct revascularization surgery for adult

\section{Study Design}

The present study was originally designed as a prospective observation study [10]. This study was carried out in accordance with the guidelines of the World Medical Association and the Declaration of Helsinki, and the protocol was reviewed and approved by the Iwate Medical University School of Medicine institutional Ethics Committee (approval No. H17-26). All patients provided written, informed consent prior to participating.

\section{Inclusion Criteria of Patients}

In total, 36 Japanese patients who visited our department from January 2008 to March 2016 and who met the following inclusion criteria were prospectively included in the original study [10]: (1) the presence of MMD according to the diagnostic criteria of the Research Committee on Spontaneous Occlusion of the Circle of Willis of the Ministry of Health, Labour and Welfare of Japan [2]; (2) age $>30$ years but $<60$ years; (3) a modified Rankin disability scale score of 0 or 1 ; $(4)$ the presence of episodes of carotid territory ischemic symptoms occurring $\leq 3$ months before presentation to our department; (5) the absence of major cerebral infarction on magnetic resonance (MR) imaging; (6) the presence of misery perfusion in the symptomatic cerebral hemispheres on brain ${ }^{15} \mathrm{O}$ gas positron emission tomography. Two patients declined surgery, and the remaining 34 patients underwent direct revascularization surgery. Two of the 34 patients were excluded following development of new ischemic lesions in the cerebral hemisphere ipsilateral to surgery as demonstrated with MR imaging, 1 day after surgery [10]. Thus, 32 patients were finally included in the original study [10].

\section{Assessment of Cognitive Changes}

Neuropsychological testing for assessment of cognitive changes was performed as described previously [10]. Briefly, each patient underwent the following neuropsychological tests: the Wechsler Adult Intelligence Scale - Revised (WAIS-R), which measures the verbal and performance intelligence quotient (IQ) [17]; the Wechsler Memory Scale (WMS), which measures the memory quotient (MQ) [18]; and the Rey-Osterrieth Complex Figure test (Rey test), which consists of copying and recalling a complex figure [19]. Cognitive abilities of the patients were thus evaluated according to scores on these 5 tests (WAIS-R verbal IQ, WAIS-R performance IQ, WMS MQ, Rey copy, and Rey recall) [10].

The neuropsychological tests were performed before surgery, 2 months after surgery [10], and at the end of the 5-year follow-up. For each neuropsychological test in each patient, a difference between scores before and after surgery (postoperative value - preoperative value) was calculated and defined as the $\Delta$ value. A significant increase was defined as a $\Delta$ value $>12.4$ for WAIS-R verbal IQ, >14.9 for WAIS-R performance IQ, >16.9 for WMS MQ, > 2.6 for Rey copy, or $>9.9$ for Rey recall [20]. A significant decrease was defined as a $\Delta$ value $<-5.6$ for WAIS-R verbal IQ,$<-5.1$ for WAIS$\mathrm{R}$ performance IQ, $<-7.5$ for WMS MQ, $<-1.8$ for Rey copy, or 
$<-4.1$ for Rey recall [20]. $\Delta$ Values outside these ranges were defined as unchanged [20]. When a patient had a significant increase in one or more neuropsychological tests, the patient was considered to have cognitive improvement [20]. When a patient had a significant decrease in one or more neuropsychological tests, the patient was considered to have cognitive decline [20]. When a patient had unchanged $\Delta$ values in all 5 neuropsychological tests, the patient was considered to have no change in cognitive function [20]. These determinations of cognitive changes were done 2 months after surgery [10] and at the end of the 5-year follow-up.

\section{Brain Perfusion Single-Photon Emission Computed}

Tomography

CBF was measured using brain perfusion single-photon emission computed tomography (SPECT) with N-isopropyl-p- $\left[{ }^{123} \mathrm{I}\right]-$ iodoamphetamine according to methods described previously [10, 21]. Patients underwent brain perfusion SPECT preoperatively, on the 1st postoperative day [10], and at the end of the 5-year followup. Postoperative cerebral hyperperfusion was defined as described previously based on brain perfusion SPECT findings before and on the day after surgery [10].

Anatomic standardization of preoperative and the 5-year follow-up SPECT images into the standard brain size and shape was performed using linear and nonlinear transformation with SPM2 [22] so that the brain images obtained from all study participants were anatomically identical. A 3-dimensional stereotaxic region of interest (ROI) template was used to set 318 constant ROIs in the cerebral and cerebellar hemispheres automatically [23]. Then, the ROIs were grouped into 10 segments (callosomarginal, pericallosal, precentral, central, parietal, angular, temporal, and posterior regions) in each hemisphere according to arterial supply, and these 10 segments were combined. The mean radioactive count on the SPECT images was measured in both cerebral and cerebellar hemispheric ROIs, and then, the mean radioactive count in the cerebral $\mathrm{ROI}$ in the symptomatic cerebral hemisphere relative to that in the ipsilateral cerebellar ROI (hemispheric relative $\mathrm{CBF}[\mathrm{RCBF}]$ ) was calculated for each patient. In addition, for each patient, the difference between hemispheric RCBF before surgery and at the end of the 5-year follow-up (postoperative value - preoperative value) was calculated and defined as $\Delta$ hemispheric RCBF.

\section{Patient Management during and after Surgery}

In all 32 patients, frontal craniotomy in the symptomatic cerebral hemisphere was performed, and the parietal branch of the superficial temporal artery was anastomosed to the M4 portion of the middle cerebral artery in the precentral region on the 3-dimensional stereotaxic ROI template because this region exhibited misery perfusion on preoperative brain ${ }^{15} \mathrm{O}$ gas positron emission tomography [10]. Cerebral hyperperfusion was diagnosed if a patient showed a major focal increase in $\mathrm{CBF}$ in the anastomosed area (the precentral region on the surgically treated side) as demonstrated by brain perfusion SPECT images the day after surgery [10]. Cerebral hyperperfusion syndrome was also diagnosed if a patient showed headache, seizures, altered consciousness, and/or new or worsening neurological symptoms, including aphasia, hemiparesis, or dysarthria between $12 \mathrm{~h}$ and 30 days postoperatively, in addition to cerebral hyperperfusion on brain perfusion SPECT [10].

Each patient received medical treatments in the outpatient setting: attempts were made to keep systolic blood pressure below 140 $\mathrm{mm} \mathrm{Hg}$, hemoglobin A1c below 6.5\%, and low-density lipoprotein cholesterol below $140 \mathrm{mg} / \mathrm{dL}$. Patients were urged to not smoke. No antiplatelet drugs were administered. MR angiography was performed before surgery and at the end of the 5-year follow-up to look for formation of collateral flow from direct revascularization.

\section{Statistical Analysis}

Data are expressed as the mean \pm standard deviation. Differences in percentages of patients with cognitive improvement, no change in cognitive function, and cognitive decline between 2 months after surgery and the end of the 5-year follow-up were evaluated using the Wilcoxon signed-rank test with continuity correction. Changes in hemispheric RCBF between preoperative status and the end of the 5-year follow-up were evaluated using the Wilcoxon signed-rank test. Differences in $\Delta$ hemispheric RCBF among patients with cognitive improvement, no change in cognitive function, and cognitive decline at the end of the 5-year followup were evaluated using Scheffe's $F$ test. Statistical significance for all analyses was set at $p<0.05$.

\section{Results}

During the 5-year follow-up period, one of the $32 \mathrm{pa}-$ tients withdrew from the present study. All of the remaining 31 patients completed neuropsychological testing and brain perfusion SPECT at the end of the 5-year follow-up.

During the 5-year follow-up, no patients experienced further ischemic events or new hemorrhagic events. Formation of collateral flow from direct revascularization was identified in all 31 patients when MR angiograms were compared before surgery and at the end of the 5-year follow-up. These comparisons of MR angiograms also showed no morphological changes in the vertebrobasilar arteries at the end of the 5-year follow-up.

Of the 31 patients analyzed, 10,8 , and 13 patients were determined to have cognitive improvement, no change in cognitive function, and cognitive decline, respectively, 2 months after surgery [10]. Figure 1 shows chronological changes in each neuropsychological test score in the 10 patients with cognitive improvement 2 months after surgery. For $\Delta$ WAIS-R verbal IQ, 2 of 6 patients with a significant increase 2 months after surgery exhibited no change, but two other patients had a new, significant increase at the end of the 5-year follow-up. For $\triangle$ WAIS-R performance IQ and $\Delta$ Rey recall, 1 patient had a new, significant increase at the end of the 5-year follow-up. For $\triangle$ WMS MQ, 1 of 2 patients with a significant increase 2 months after surgery exhibited no change, but 3 other patients had a new, significant increase at the end of the 5 -year follow-up. For $\Delta$ Rey copy, 2 patients had a new, significant increase at the end of the 5-year follow-up. Therefore, of 10 patients with cognitive improvement 2 


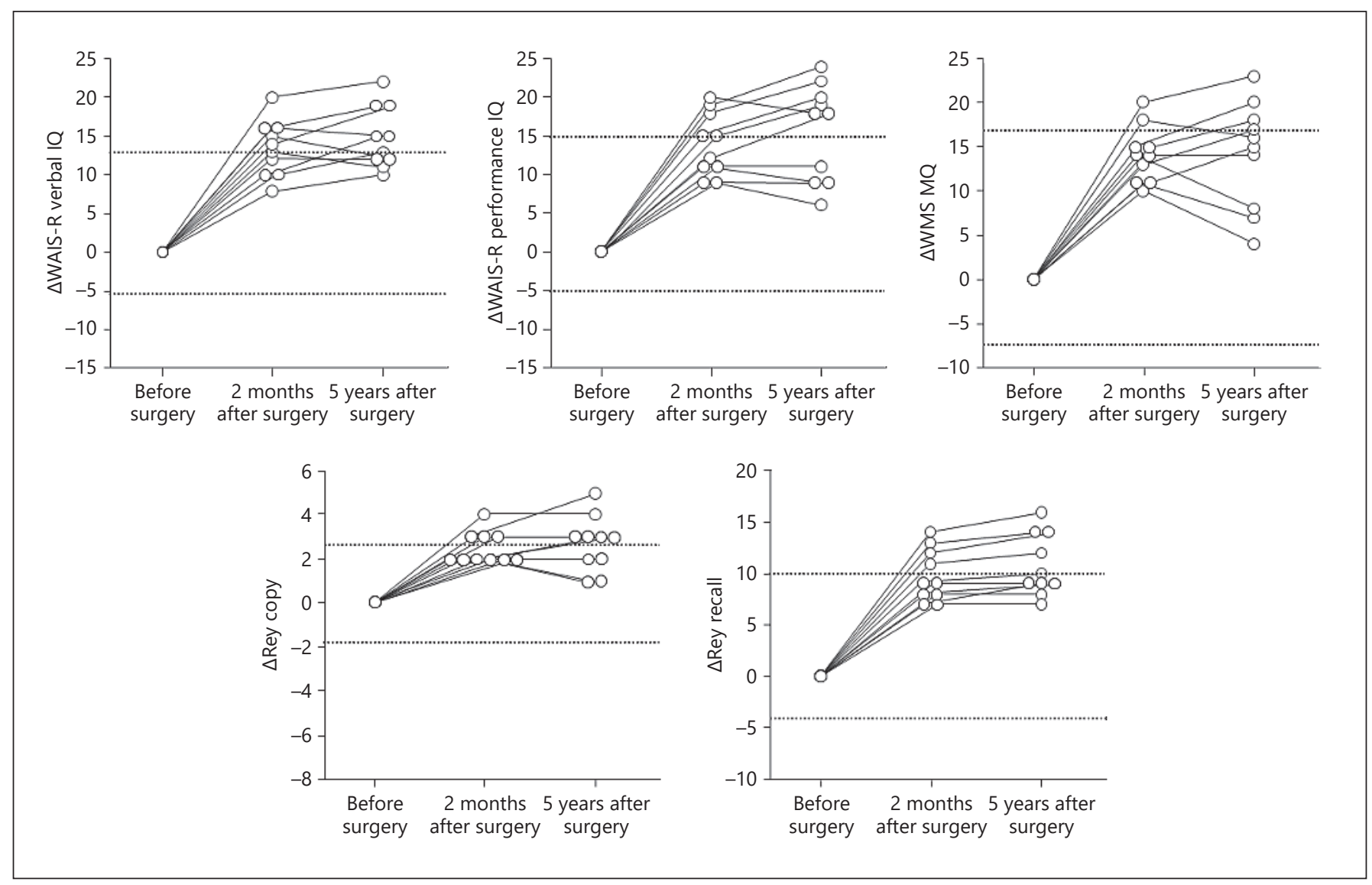

Fig. 1. Chronological changes in $\Delta$ value of each neuropsychological test score in 10 patients with cognitive improvement 2 months after surgery. Upper and lower dotted lines indicate cutoff points of a significant increase and decrease, respectively, for each neuropsychological test score.

months after surgery, one exhibited no change in cognitive function, and 9 retained cognitive improvement at the end of the 5-year follow-up.

Figure 2 shows chronological changes in each neuropsychological test in the 8 patients without a change in cognitive function 2 months after surgery. For $\triangle$ WAIS-R verbal IQ and $\Delta$ Rey recall, 1 patient had a new, significant increase at the end of the 5 -year follow-up. For $\triangle$ WAIS-R performance IQ, $\triangle \mathrm{WMS} \mathrm{MQ}$, and $\Delta$ Rey copy, all 8 patients remained unchanged at the end of the 5-year follow-up. Therefore, of 8 patients without a change in cognitive function 2 months after surgery, 2 exhibited cognitive improvement, and 6 remained cognitively unchanged at the end of the 5-year follow-up.

Figure 3 shows chronological changes in each neuropsychological test score in 13 patients with cognitive decline 2 months after surgery. For $\Delta$ WAIS-R verbal IQ, 4 of 7 patients with a significant decrease 2 months after surgery exhibited no change, but another patient had a new, significant decrease at the end of the 5-year followup. For $\Delta$ WAIS-R performance IQ, 1 of 6 patients with a significant decrease 2 months after surgery exhibited no change. For $\triangle \mathrm{WMS} M \mathrm{MQ}, 1$ patient with a significant decrease 2 months after surgery exhibited no change, but 2 other patients had a new, significant decrease at the end of the 5 -year follow-up. For $\Delta$ Rey copy, 2 patients had a new, significant decrease at the end of the 5-year followup. For $\Delta$ Rey recall, 1 patient with a significant decrease 2 months after surgery exhibited no change at the end of the 5-year follow-up. Therefore, of 13 patients with cognitive decline 2 months after surgery, 3 exhibited no change in cognitive function, and 10 continued to show cognitive impairment at the end of the 5-year follow-up. Seven of 9 patients with cerebral hyperperfusion syn- 


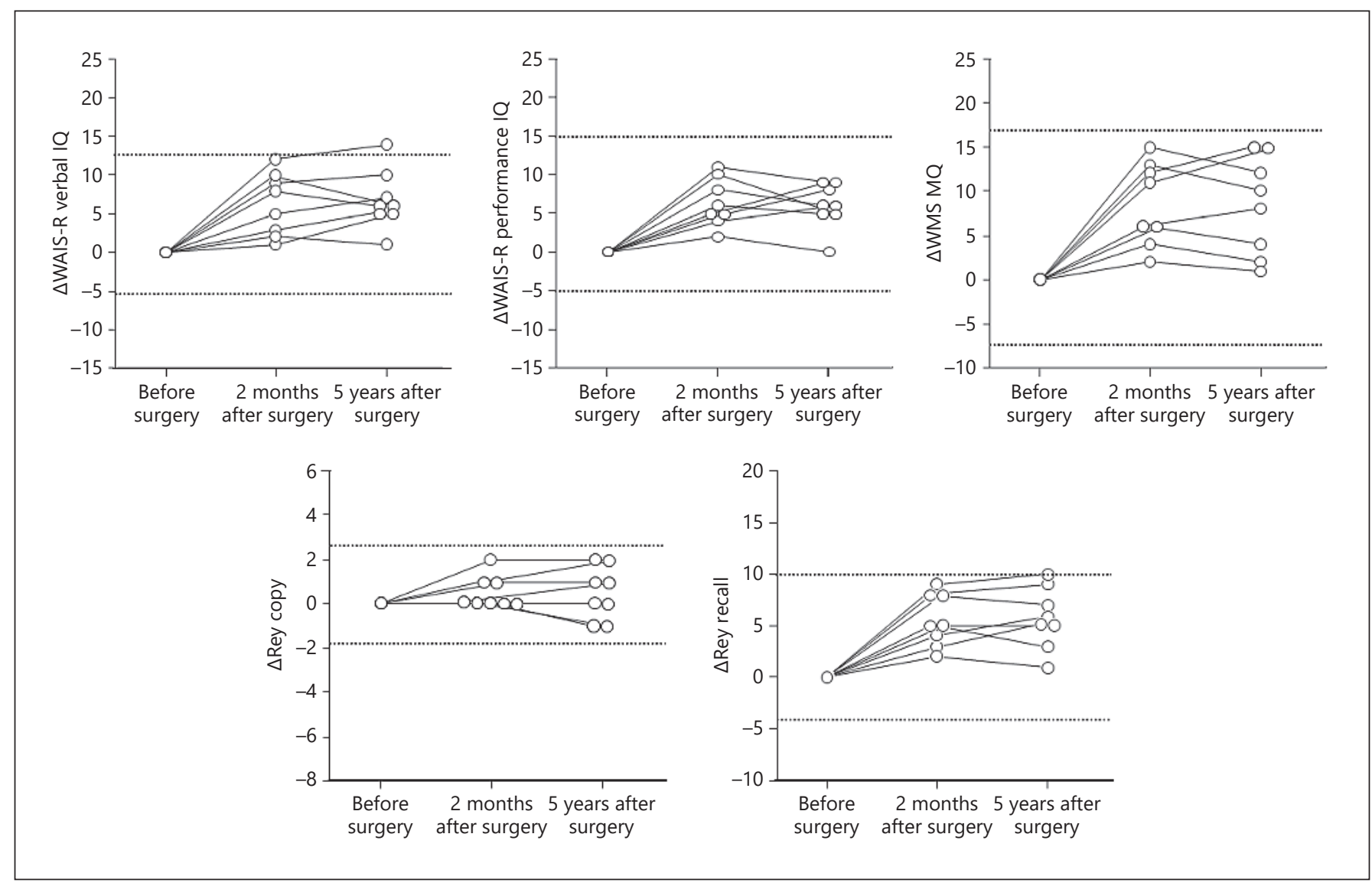

Fig. 2. Chronological changes in $\Delta$ value of each neuropsychological test score in 8 patients without a change in cognitive function 2 months after surgery. Upper and lower dotted lines indicate cutoff points of a significant increase and decrease, respectively, for each neuropsychological test score.

drome after surgery continued to show cognitive impairment even at the end of the 5-year follow-up. Three other patients with cognitive decline at the end of the 5-year follow-up had asymptomatic cerebral hyperperfusion on brain perfusion SPECT on the day after surgery.

Based on the above results, 11,10 , and 10 patients were considered to have cognitive improvement, no change in cognitive function, and cognitive decline, respectively, at the end of the 5-year follow-up. These ratios were not significantly different compared with those 2 months after surgery.

Figure 4 shows changes in hemispheric RCBF in patients with cognitive improvement, no change in cognitive function, and cognitive decline at the end of the 5 -year follow-up. The hemispheric RCBF was significantly greater at the end of the 5-year follow-up than before surgery in patients with cognitive improvement (80.7 \pm
$6.1 \%$ vs. $92.9 \pm 5.5 \%$; $p=0.0033$ ) or no change in cognitive function $(85.6 \pm 3.5$ vs. $91.5 \pm 5.2 \%$; $p=0.0093)$. This value was significantly lower at the end of the 5 -year follow-up than before surgery in patients with cognitive decline $(83.8 \pm 3.7$ vs. $81.0 \pm 5.8 \% ; p=0.0367)$. $\Delta$ Hemispheric RCBF differed significantly among these 3 patient groups (cognitive improvement vs. no change in cognitive function, $p=0.0019$; no change in cognitive function vs. cognitive decline, $p<0.0001$; and cognitive decline vs. cognitive improvement, $p<0.0001$ ) and was, in the order of descending prevalence, cognitive improvement (12.1 \pm $3.1)$, no change in cognitive function $(5.9 \pm 4.3)$, and cognitive decline $(-2.8 \pm 3.5)$. Figures 5 and 6 show MR angiograms and brain perfusion SPECT images in a patient with cognitive improvement and in a patient with cognitive decline at the end of the 5-year follow-up, respectively. 


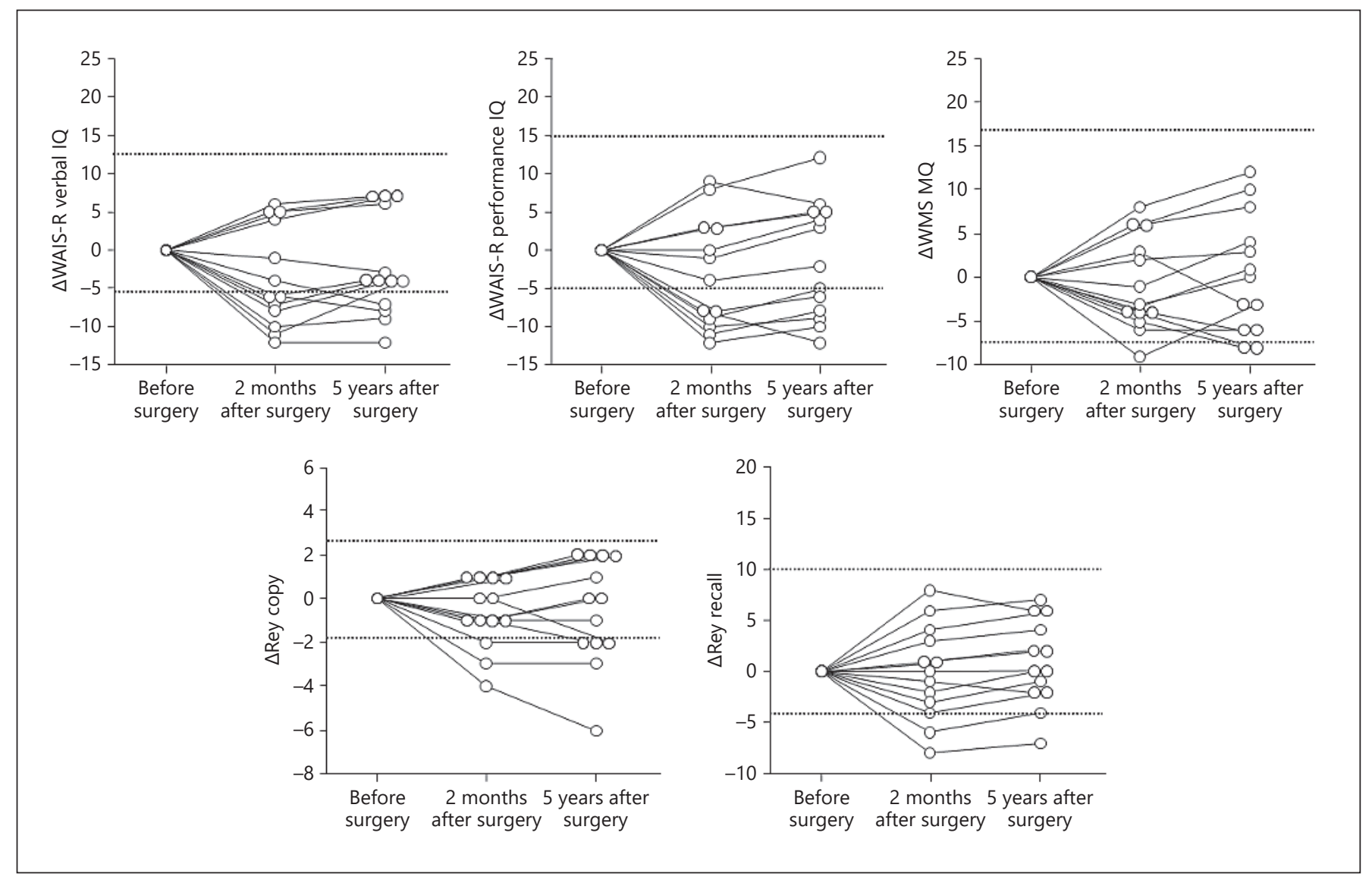

Fig. 3. Chronological changes in $\Delta$ value of each neuropsychological test score in 13 patients with cognitive decline 2 months after surgery. Upper and lower dotted lines indicate cutoff points of a significant increase and decrease, respectively, for each neuropsychological test score.

Fig. 4. Changes in hemispheric RCBF in patients with cognitive improvement, no change in cognitive function, and cognitive decline at the end of the 5-year follow-up.

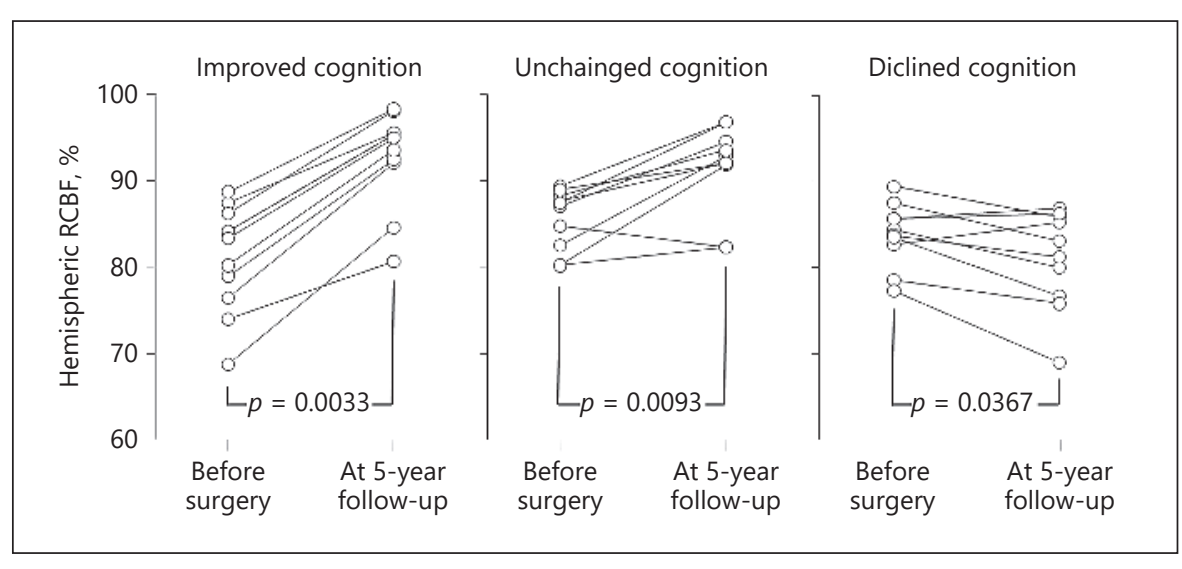

\section{Discussion}

The present prospective study demonstrated that onethird of adult patients with cerebral misery perfusion due to ischemic MMD who underwent direct revasculariza- tion surgery exhibited cognitive improvement, and onethird had cognitive decline at the end of the 5-year followup. The results also indicated that the former and latter patients had increased and decreased CBF, respectively, in the affected cerebral hemisphere at the end of the 


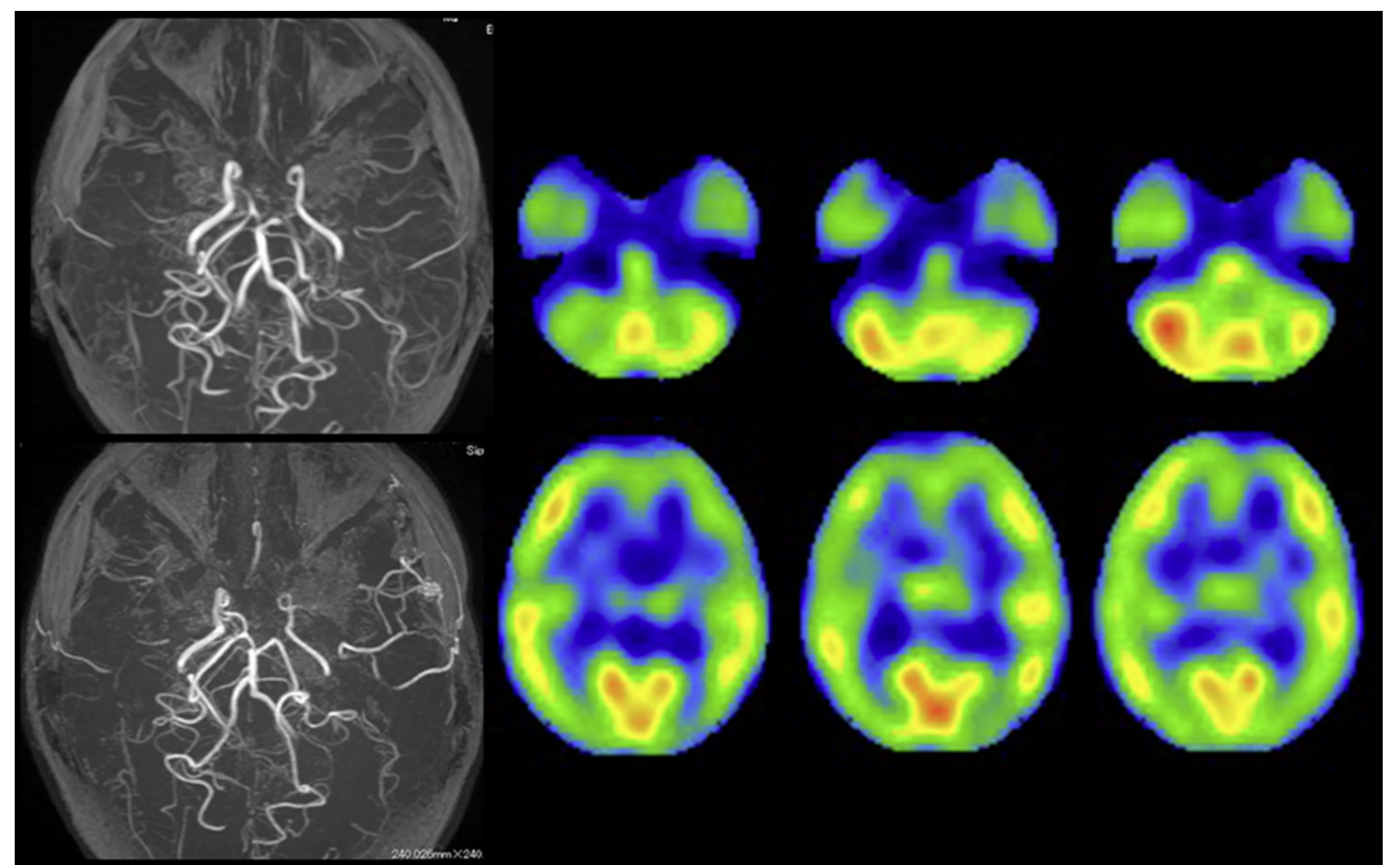

Fig. 5. A 35-year-old woman with ischemic MMD who underwent direct revascularization surgery in the left cerebral hemisphere and exhibited improved cognition at the end of the 5-year follow-up period. Formation of collateral flow from direct revascularization is identified when MR angiograms are compared before surgery (left end, upper) and at the end of the 5-year follow-up (left end, lower). Blood flow in the left frontal lobe is decreased relative to that in the left cerebellar hemisphere on brain perfusion SPECT before surgery (second from the left). The blood flow is increased on the day after surgery (second from the right), and this increased blood flow is maintained at the end of the 5-year follow-up period (right, end). 5-year follow-up compared with preoperative brain perfusion.

Several retrospective studies reported the incidence of further ischemic or new hemorrhagic events after direct revascularization surgery in adult patients with ischemic MMD: 8\% for 56.2 months [24], 0\% for 38.2 months [25], and $<0.2 \%$ for 60 months [26]. Our results showing no further ischemic events or new hemorrhagic events during the 5-year follow-up were comparable with these previous findings.

In the present study, cognitive function in 6 patients changed at the end of the 5-year follow-up compared with that of 2 months after surgery. However, the percentages of patients with cognitive improvement, no change in cognitive function, and cognitive decline did not change, and one-third of adult patients with cerebral misery perfusion due to ischemic MMD who underwent direct revascularization surgery exhibited cognitive improvement, and one-third had cognitive decline over the long term. In particular, most patients with cerebral hyperperfusion syndrome after surgery continued to show cognitive dysfunction even at the end of the 5-year follow-up. A case of an adult MMD patient with the same clinical condition was reported: this patient developed cognitive decline accompanied by brain atrophy due to cerebral hyperperfusion syndrome after direct revascularization surgery [27]. Further, a study using animal models demonstrated that cerebral hyperperfusion following reperfusion after 3 days of hemispheric hypoperfusion induces white matter injury, inflammation, and subsequent cognitive decline as well as blood-brain barrier breakdown [28]. Therefore, postoperative cerebral hyperperfusion may produce wide- 


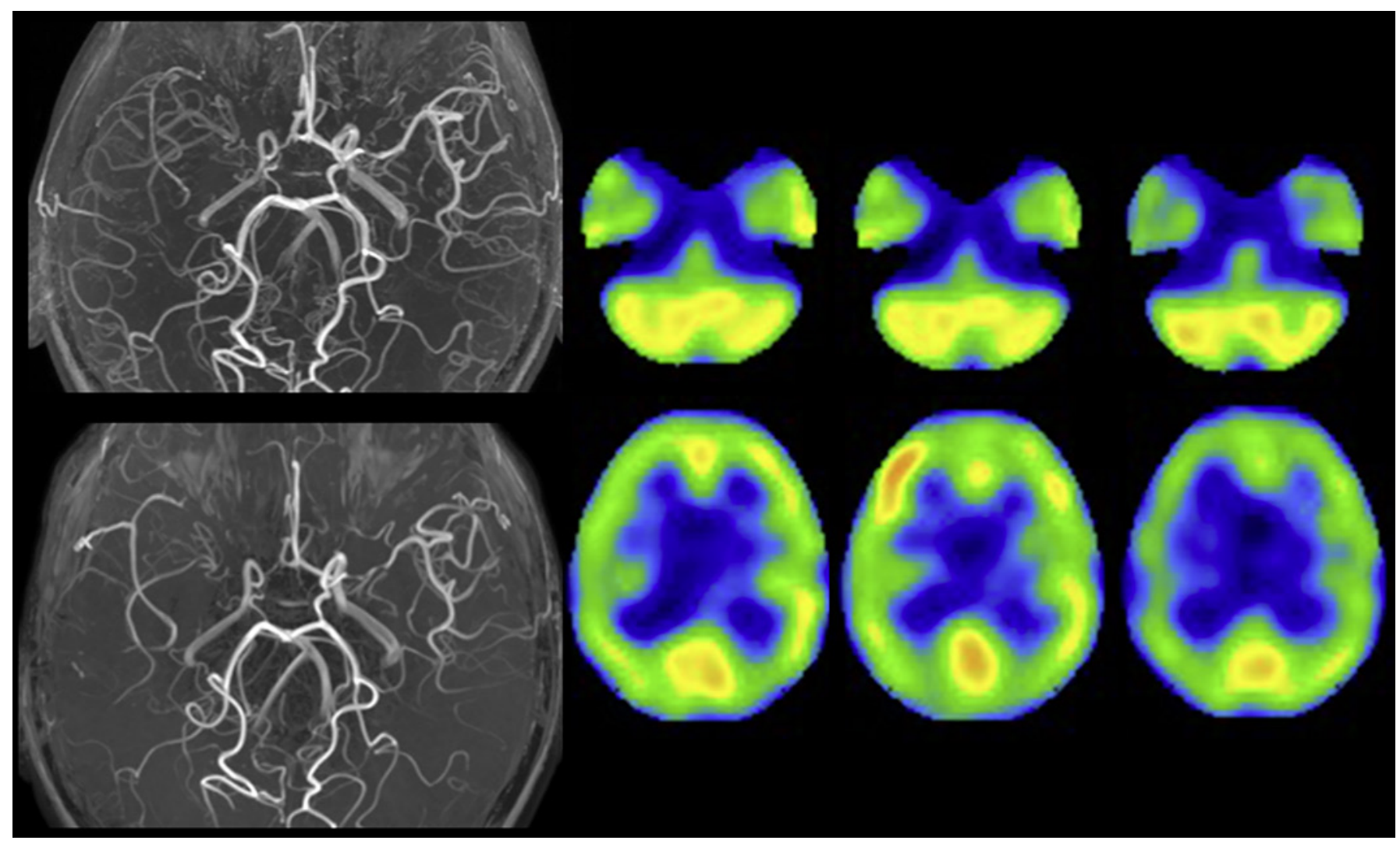

Fig. 6. A 44-year-old woman with ischemic MMD who underwent direct revascularization surgery in the right cerebral hemisphere and exhibited cognitive decline at the end of the 5-year follow-up period. Formation of collateral flow from direct revascularization is identified when MR angiograms are compared before surgery (left end, upper) and at the end of the 5-year follow-up (left end, lower). Blood flow in the right frontal lobe is decreased relative to that in the left cerebellar hemisphere on brain perfusion SPECT before surgery (second from the left). Hyperperfusion develops in the right frontal lobe on the day after surgery (second from the right), and blood flow in the right cerebral hemisphere is decreased more prominently at the end of the 5-year follow-up (right, end) than before surgery. spread damage to the ipsilateral cerebral hemisphere, resulting in irreversible cognitive decline.

Another important result of our study was an increase in CBF in the affected cerebral hemisphere in patients who showed cognitive improvement at 5 years. Previous studies have demonstrated improved cerebral oxygen metabolism along with increased $\mathrm{CBF}$ after direct revascularization surgery in the cerebral hemisphere with misery perfusion caused by MMD [29]. This improved metabolism is accompanied by improved postsurgical cognitive function [11]. Our observations are consistent with these previous reports and suggest that improvements in metabolism may be retained for a long time. On the other hand, $\mathrm{CBF}$ in most patients with reduced cognitive function was under the preoperative value 5 years later. Two pathophysiologically distinct conditions may result from reduced CBF: misery perfusion as a result of hemo- dynamic compromise and matched hypometabolism [30]. A prospective cohort study was performed that enrolled adult patients with ischemic MMD who were treated with medication only. Results showed that upon development of subsequent ischemic events, the affected cerebral hemisphere shows new misery perfusion, and patients without misery perfusion do not experience ischemic events. Thus, new development of misery perfusion may predict additional ischemic events in adult patients with ischemic MMD [3, 4]. None of the patients in our current study who exhibited reduced CBF at the end of the 5-year follow-up exhibited additional ischemic events and thus may not show misery perfusion. In contrast, for matched hypometabolism, damage to neurons leads to decreased metabolism in the cerebral cortex even if the cortex appears normal [31]. In this situation, $\mathrm{CBF}$ and cerebral metabolism are decreased in parallel [31]. Therefore, de- 
creased CBF at the end of the 5-year follow-up may indicate decreased cerebral metabolism, which leads to decreased cognitive function. In such patients, CBF 2 months after surgery was higher than the presurgical value [10], and CBF may gradually decrease over 5 years along with a decrease in cerebral metabolism.

\section{Conclusion}

One-third of adult patients with cerebral misery perfusion due to ischemic MMD who underwent direct revascularization surgery exhibited cognitive improvement, and one-third patients exhibited decline at the end of the 5-year follow-up. The results also indicated that the former and latter patients had increased and decreased CBF, respectively, in the affected cerebral hemisphere at the end of the 5-year follow-up compared with preoperative brain perfusion.

\section{Statement of Ethics}

This prospective observational study was carried out in accordance with the guidelines of the World Medical Association and Declaration of Helsinki, and the protocol was reviewed and approved by the Iwate Medical University School of Medicine institutional Ethics Committee (No. H17-26). Written, informed consent was obtained from all patients or their next of kin before the study began.

\section{Conflict of Interest Statement}

The author (Kuniaki Ogasawara) declared the following potential conflicts of interest with respect to the research, authorship, and/or publication of this article: consigned research funds from
Nihon Medi-Physics Co., Ltd. The corresponding author (Kuniaki Ogasawara) is one of the present journal's editorial board members.

\section{Funding Sources}

This study was supported in part by Grants-in-Aid for Scientific Research from the Japan Society for the Promotion of Science (21K09108 and 21K09157).

\section{Author Contributions}

Shun Uchida and Kuniaki Ogasawara contributed to conception and design, acquisition of data, analysis and interpretation of data, drafting the article critically for important intellectual content, and the final approval of the version to be published. Yoshitaka Kubo, Masahiro Yabuki, Shunrou Fujiwara, Kenji Yoshida, and Masakazu Kobayashi contributed to conception and design, revising the article critically for important intellectual content, and the final approval of the version to be published. Daisuke Oomori, Kei Kitakami, and Kazunori Terasaki contributed to acquisition of data, revising the article critically for important intellectual content, and the final approval of the version to be published. All the authors agree to be accountable for all aspects of the work in ensuring that questions related to the accuracy or integrity of any part of the work are appropriately investigated and resolved.

\section{Data Availability Statement}

All data generated or analyzed during this study are not publicly available on ethical grounds. However, inquiries regarding these data can be directed to the corresponding author.

\section{References}

1 Suzuki J, Takaku A. Cerebrovascular "moyamoya" disease. Disease showing abnormal net-like vessels in base of brain. Arch Neurol. 1969;20:288-99.

2 Research Committee on the Pathology and Treatment of Spontaneous Occlusion of the Circle of Willis. Guidelines for diagnosis and treatment of moyamoya disease (spontaneous occlusion of the circle of Willis). Neurol Med Chir. 2012;52:245-66.

3 Miyoshi K, Chida K, Kobayashi M, Kubo Y, Yoshida K, Terasaki K, et al. Two-year clinical, cerebral hemodynamic and cognitive outcomes of adult patients undergoing medication alone for symptomatically ischemic moyamoya disease without cerebral misery perfusion: a prospective cohort study. Neurosurgery. 2018;84:1233-41.
4 Kitakami K, Kubo Y, Yabuki M, Oomori D, Takahashi T, Igarashi S, et al. Five-year outcomes of medical management alone for adult patients with ischemic moyamoya disease without cerebral misery perfusion. Cerebrovasc Dis. 2021:1-7. Epub ahead of print.

5 Seo WK, Kim JY, Choi EH, Kim YS, Chung JW, Saver JL, et al. Association of antiplatelet therapy, including cilostazol, with improved survival in patients with moyamoya disease in a nationwide study. J Am Heart Assoc. 2021; 10:e017701.

6 Fujimura M, Tominaga T. Current status of revascularization surgery for moyamoya disease: special consideration for its 'internal carotid-external carotid (IC-EC) conversion' as the physiological reorganization system. Tohoku J Exp Med. 2015;236:45-53.
7 Guzman R, Lee M, Achrol A, Stephens TB, Kelly M, Do HM, et al. Clinical outcome after 450 revascularization procedures for moyamoya disease. J Neurosurg. 2009;111:927-35.

8 Narisawa A, Fujimura M, Tominaga T. Efficacy of the revascularization surgery for adult-onset moyamoya disease with the progression of cerebrovascular lesions. Clin Neurol Neurosurg. 2009;111:123-6.

9 Nama KW, Chob WS, Kwon HM, Kim JE, Lee YS, Park SW, et al. Ivy sign predicts ischemic stroke recurrence in adult moyamoya patients without revascularization surgery. Cerebrovasc Dis. 2019;47:223-30.
Cognition after Revascularization in Moyamoya Disease
Cerebrovasc Dis Extra 2021;11:145-154 DOI: $10.1159 / 000521028$ 
10 Yanagihara W, Chida K, Kobayashi M, Kubo Y, Yoshida K, Terasaki K, et al. Impact of cerebral blood flow changes due to arterial bypass surgery on cognitive function in adult patients with symptomatic ischemic moyamoya disease. J Neurosurg. 2018;131:1716-24.

11 Ando T, Shimada Y, Fujiwara S, Yoshida K, Kobayashi M, Kubo Y, et al. Revascularisation surgery improves cognition in adult patients with moyamoya disease. J Neurol Neurosurg Psychiatry. 2020;91:332-4.

12 Kazumata K, Tha KK, Tokairin K, Ito M, Uchino $\mathrm{H}$, Kawabori M, et al. Brain structure, connectivity, and cognitive changes following revascularization surgery in adult moyamoya disease. Neurosurgery. 2019;85:E943-E52.

13 Calviere L, Loubiere P, Planton M, Cazzola V, Catalaa I, Mirabel H, et al. Decreased frontal white-matter diffusion and improved cognitive flexibility after burr-hole surgery in moyamoya angiopathy. BMC Neurol. 2020;20:30.

14 Hara S, Kudo T, Hayashi S, Inaji M, Tanaka $\mathrm{Y}$, Maehara $\mathrm{T}$, et al. Improvement in cognitive decline after indirect bypass surgery in adult moyamoya disease: implication of $15 \mathrm{O}$-gas positron emission tomography. Ann Nucl Med. 2020;34:467-75.

15 Lei Y, Li YJ, Guo QH, Liu XD, Liu Z, Ni W, et al. Postoperative executive function in adult moyamoya disease: a preliminary study of its functional anatomy and behavioral correlates. J Neurosurg. 2017;126:527-36.

16 Zeifert PD, Karzmark P, Bell-Stephens TE, Steinberg GK, Dorfman LJ. Neurocognitive performance after cerebral revascularization in adult moyamoya disease. Stroke. 2017;48: $1514-7$.
17 Shinagawa F, Kobayashi S, Fujita K. Japanese wechsler adult intelligence scale-revised. Tokyo: Nihon Bunka Kagakusha; 1990.

18 Koyama M. Japanese wechsler memory scale. In: Koyama M, editor. Clinical psychology of brain damage. Tokyo: Gakuen Sha; 1985. p. 48-54.

19 Lezak MD. Neuropsychological assessment. 3rd ed. New York: Oxford University Press; 1995.

20 Yoshida K, Ogasawara K, Kobayashi M, Yoshida K, Kubo Y, Otawara Y, et al. Improvement and impairment in cognitive function after carotid endarterectomy: comparison of objective and subjective assessments. Neurol Med Chir. 2012;52:154-60.

21 Setta K, Kojima D, Shimada Y, Yoshida J, Oshida S, Fujimoto K, et al. Accuracy of brain perfusion single-photon emission computed tomography for detecting misery perfusion in adult patients with symptomatic ischemic moyamoya disease. Ann Nucl Med. 2018;32: 611-9.

22 Nishimiya M, Matsuda H, Imabayashi E, Kuji I, Sato N. Comparison of SPM and NEUROSTAT in voxelwise statistical analysis of brain SPECT and MRI at the early stage of Alzheimer's disease. Ann Nucl Med. 2008;22:921-7.

23 Takeuchi R, Matsuda H, Yoshioka K, Yonekura Y. Cerebral blood flow SPET in transient global amnesia with automated ROI analysis by 3DSRT. Eur J Nucl Med Mol Imaging. 2004;31:578-89.

24 Lee SB, Kim DS, Huh PW, Yoo DS, Lee TG, Cho KS. Long-term follow-up results in 142 adult patients with moyamoya disease according to management modality. Acta Neurochir. 2012;154:1179-87.
25 Kraemer M, Karakaya R, Matsushige T, Graf J, Albrecht P, Hartung HP, et al. Efficacy of STA-MCA bypass surgery in moyamoya angiopathy: long-term follow-up of the Caucasian Krupp Hospital cohort with 81 procedures. J Neurol. 2018;265:2425-33.

26 Lai PMR, Patel NJ, Frerichs KU, Patel AB, Aziz-Sultan MA, Ogilvy CS, et al. Direct vs indirect revascularization in a North American Cohort of moyamoya disease. Neurosurgery. 2021;89:315-22.

27 Ogasawara K, Komoribayashi N, Kobayashi $\mathrm{M}$, Fukuda $\mathrm{T}$, Inoue $\mathrm{T}$, Yamadate $\mathrm{K}$, et al. Neural damage caused by cerebral hyperperfusion after arterial bypass surgery in a patient with moyamoya disease: case report. Neurosurgery. 2005;56:E1380.

28 Mansour A, Rashad S, Niizuma K, Fujimura M, Tominaga T. A novel model of cerebral hyperperfusion with blood-brain barrier breakdown, white matter injury, and cognitive dysfunction. J Neurosurg. 2020;133(5): 1460-72.

29 Kuroda S, Kashiwazaki D, Hirata K, Shiga T, Houkin K, Tamaki N. Effects of surgical revascularization on cerebral oxygen metabolism in patients with moyamoya disease: an 15O-gas positron emission tomographic study. Stroke. 2014;45:2717-21.

30 Kuroda S, Shiga T, Houkin K, Ishikawa T, Katoh C, Tamaki N, et al. Cerebral oxygen metabolism and neuronal integrity in patients with impaired vasoreactivity attributable to occlusive carotid artery disease. Stroke. 2006; 37:393-8.

31 Yamauchi H, Nishii R, Higashi T, Kagawa S, Fukuyama H. Silent cortical neuronal damage in atherosclerotic disease of the major cerebral arteries. J Cereb Blood Flow Metab. 2011; 31:953-61. 